\title{
A modified interval symmetric single step procedure ISS-5D for simultaneous inclusion of polynomial zeros
}

\begin{abstract}
The aim of this paper is to present a new modified interval symmetric single-step procedure ISS-5D which is the extension from the previous procedure, ISS1. The ISS-5D method will produce successively smaller intervals that are guaranteed to still contain the zeros. The efficiency of this method is measured on the CPU times and the number of iteration. The procedure is run on five test polynomials and the results obtained are shown in this paper.
\end{abstract}

Keyword: Analysis interval; CPU times; Zero of a polynomial 